\title{
Supporting Information: Solution properties of polyelectrolytes with divalent counterions
}

\author{
Carlos G. Lopez, ${ }^{1, \text { a) }}$ Ferenc Horkay, ${ }^{2}$ Ralf Schweins, ${ }^{3}$ and Walter Richtering ${ }^{4}$ \\ 1) Institute of Physical Chemistry, RWTH Aachen University, Landoltweg 2, 52056 Aachen, \\ Germany \\ ${ }^{2)}$ Section on Quantitative Imaging and Tissue Sciences, Eunice Kennedy Shriver National \\ Institute of Child Health and Human Development, 13 South Drive, Bethesda, Maryland 20892, \\ USA \\ ${ }^{3)}$ Institut Laue-Langevin DS/LSS, 71 avenue des Martyrs, CS 20156, 38042 Grenoble Cedex 9, \\ France \\ ${ }^{4)}$ Institute of Physical Chemistry, RWTH Aachen University, Landoltweg 2, 52056 Aachen, \\ Germany
}

Figure 1 shows fits to Eq. 3 of the main text, the best fit values for the effective monomer length are $b^{\prime} \simeq$ $0.26-0.27 \mathrm{~nm}$ for all samples except for the the red curve in the bottom panel, which gives a lower value of $\simeq 0.24$ nm.

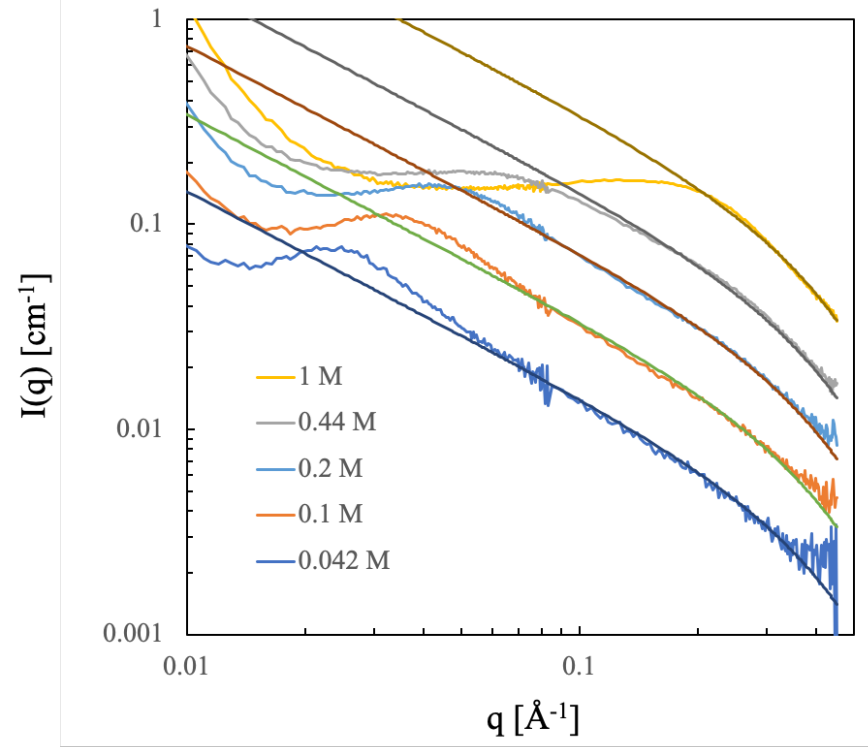

FIG. 1. Fits to the WLC model at high q for semidilute solutions of MgPSS with $N=780$ in salt-free $\mathrm{D}_{2} \mathrm{O}$. Polymer concentrations are indicated on the legend. Data are measured at D11.

Figure 2 shows fits to the high-q WLC form factor for NaPSS in excess $\mathrm{NaCl} \mathrm{D}_{2} \mathrm{O}$ solution. The polymer concentrations are indicated on the caption of the figure.

The effective monomer size for NaPSS measured by SANS from different studies is compiled in table I. Values are higher than the value estimated from the correlation peak $b^{\prime}=b / B \simeq 0.17 \mathrm{~nm}$ with $B=1.5$.
TABLE I. Effective monomer size for NaPSS from different literature sources

\begin{tabular}{lc}
$b^{\prime}[\mathrm{nm}]$ & Reference \\
\hline $0.21-0.24$ & This work \\
0.17 & {$[1]$} \\
0.22 & {$[2]$} \\
0.25 & {$[3]$} \\
0.2 & {$[4]$}
\end{tabular}

\section{VISCOSITY AS A FUNCTION OF SHEAR RATE}

Figure 3 shows the viscosity of CaPSS with $M_{w} \simeq$ 150 in DI water at different polymer concentrations as a function of shear rate. The samples can be seen to be essentially Newtonian.

For higher molar masses and high concentrations, shear thinning can be observed. The top panel of figure 4 shows the shear rate dependence of the viscosity of MgPSS at high concentrations $(0.72 \mathrm{M}$ and $1 \mathrm{M})$. The bottom panel of the same figure plots the inverse of the apparent viscosity as a function of the square root of the shear rate, which yield approximately linear curves. This can be then be used to extrapolate the zero-shear rate viscosity. The error in such estimation is of the order of $15-20 \%$, which is of the order of the symbol size in Figs 3,4 and 6 of the main text.

\section{REFERENCES}

${ }^{1}$ K. Kassapidou, W. Jesse, M. Kuil, A. Lapp, S. Egelhaaf, and J. Van der Maarel, Macromolecules 30, 2671 (1997).

${ }^{2}$ F. Boué, J. Cotton, A. Lapp, and G. Jannink, The Journal of chemical physics 101, 2562 (1994).

${ }^{3}$ M. Spiteri, F. Boué, A. Lapp, and J. Cotton, Physical review letters 77, 5218 (1996).

${ }^{4}$ J. Combet, P. Lorchat, and M. Rawiso, The European Physical Journal Special Topics 213, 243 (2012). 

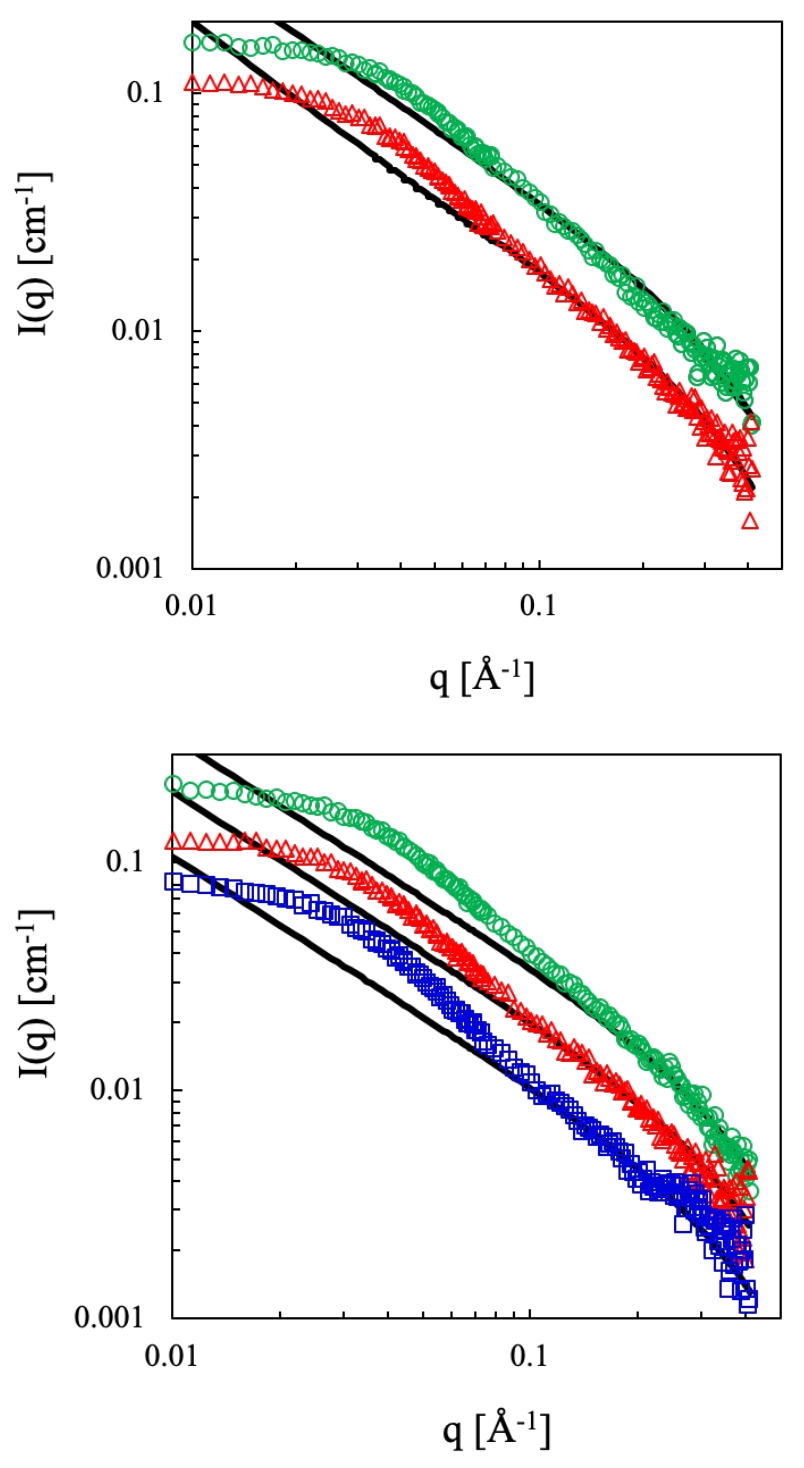

FIG. 2. Fits to the WLC model at high q for semidilute solutions of NaPSS with $\mathrm{Mw}=29.1 \mathrm{~kg} / \mathrm{mol}$ in $0.375 \mathrm{M} \mathrm{NaCl}$ (top panel) and $0.75 \mathrm{M} \mathrm{NaCl}$ (bottom panel). Polymer concentrations: Blue: 0.027M, red: 0.045M, green: 0.9M. Black lines are fits to the WLC at high q. Values of $b^{\prime}$ are given in Table I 


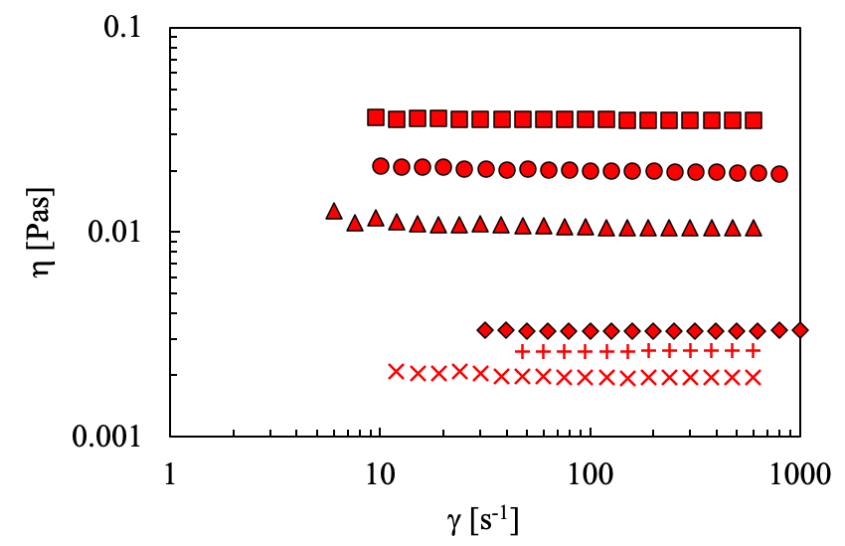

FIG. 3. Apparent viscosity as a function of shear rate for CaPSS with $M_{w}=150 \mathrm{~kg} / \mathrm{mol}$.
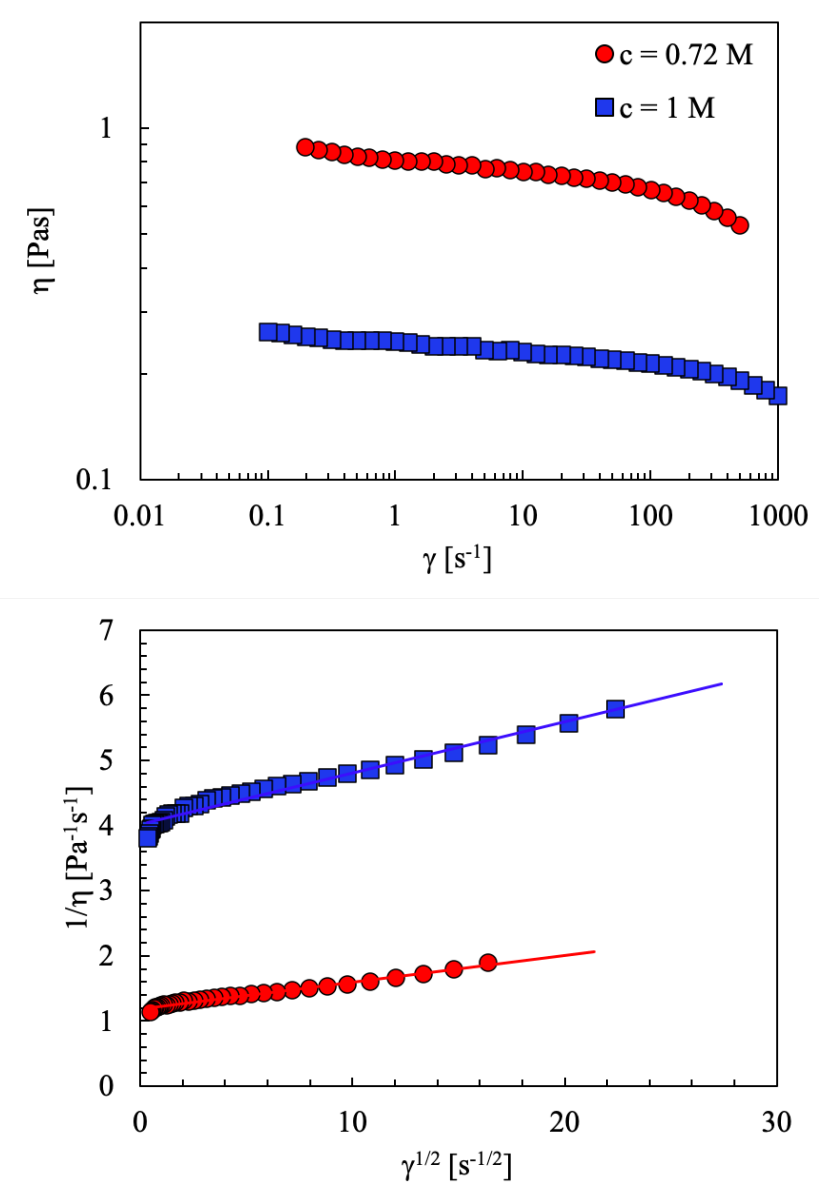

FIG. 4. Apparent viscosity of two MgPSS samples with $M_{w} \simeq$ $1 \mathrm{M}$ as a function of shear rate and extrapolation procedure for zero shear-rate value. 
TABLE II. Viscosity Data

\begin{tabular}{|c|c|c|c|c|c|}
\hline $\mathrm{Mw}[\mathrm{g} / \mathrm{mol}]$ & $\mathrm{c}[\mathrm{M}]$ & $\eta_{s p}[-]$ & $\mathrm{Mw}[\mathrm{g} / \mathrm{mol}]$ & $\mathrm{c}[\mathrm{M}]$ & $\eta_{s p}[-]$ \\
\hline $10 \mathrm{k}$ & $2.49 \mathrm{E}+00$ & $2.78 \mathrm{E}+02$ & $271 \mathrm{k}$ & $6.42 \mathrm{E}-01$ & $3.18 \mathrm{E}+01$ \\
\hline $10 \mathrm{k}$ & $1.76 \mathrm{E}+00$ & $1.74 \mathrm{E}+01$ & $271 \mathrm{k}$ & $5.29 \mathrm{E}-01$ & $2.32 \mathrm{E}+01$ \\
\hline $10 \mathrm{k}$ & $1.14 \mathrm{E}+00$ & $4.01 \mathrm{E}+00$ & $271 \mathrm{k}$ & $3.50 \mathrm{E}-01$ & $1.23 \mathrm{E}+01$ \\
\hline $10 \mathrm{k}$ & $9.85 \mathrm{E}-01$ & $2.99 \mathrm{E}+00$ & $271 \mathrm{k}$ & $2.32 \mathrm{E}-01$ & $7.57 \mathrm{E}+00$ \\
\hline $10 \mathrm{k}$ & 7.31E-01 & $1.55 \mathrm{E}+00$ & $271 \mathrm{k}$ & $1.54 \mathrm{E}-01$ & $5.13 \mathrm{E}+00$ \\
\hline $15 \mathrm{k}$ & $2.48 \mathrm{E}+00$ & $4.54 \mathrm{E}+02$ & $271 \mathrm{k}$ & $1.27 \mathrm{E}-01$ & $4.36 \mathrm{E}+00$ \\
\hline $15 \mathrm{k}$ & $1.74 \mathrm{E}+00$ & $2.60 \mathrm{E}+01$ & $271 \mathrm{k}$ & $8.54 \mathrm{E}-02$ & $3.17 \mathrm{E}+00$ \\
\hline $15 \mathrm{k}$ & $1.23 \mathrm{E}+00$ & $6.69 \mathrm{E}+00$ & $271 \mathrm{k}$ & $5.59 \mathrm{E}-02$ & $2.38 \mathrm{E}+00$ \\
\hline $15 \mathrm{k}$ & $9.94 \mathrm{E}-01$ & $3.99 \mathrm{E}+00$ & $271 \mathrm{k}$ & $4.08 \mathrm{E}-02$ & $1.96 \mathrm{E}+00$ \\
\hline $15 \mathrm{k}$ & $7.66 \mathrm{E}-01$ & $2.36 \mathrm{E}+00$ & $271 \mathrm{k}$ & $2.83 \mathrm{E}-02$ & $1.57 \mathrm{E}+00$ \\
\hline $15 \mathrm{k}$ & $6.06 \mathrm{E}-01$ & $1.64 \mathrm{E}+00$ & $271 \mathrm{k}$ & $1.94 \mathrm{E}-02$ & $1.24 \mathrm{E}+00$ \\
\hline $30 \mathrm{k}$ & $2.49 \mathrm{E}+00$ & $6.96 \mathrm{E}+02$ & $271 \mathrm{k}$ & $1.37 \mathrm{E}-02$ & $9.89 \mathrm{E}-01$ \\
\hline $30 \mathrm{k}$ & $1.98 \mathrm{E}+00$ & $7.88 \mathrm{E}+01$ & $480 \mathrm{k}$ & $1.23 \mathrm{E}+00$ & $5.05 \mathrm{E}+02$ \\
\hline $30 \mathrm{k}$ & $1.69 \mathrm{E}+00$ & $3.54 \mathrm{E}+01$ & $480 \mathrm{k}$ & $9.99 \mathrm{E}-01$ & $2.71 \mathrm{E}+02$ \\
\hline $30 \mathrm{k}$ & $1.24 \mathrm{E}+00$ & $1.08 \mathrm{E}+01$ & $480 \mathrm{k}$ & 7.93E-01 & $1.43 \mathrm{E}+02$ \\
\hline $30 \mathrm{k}$ & $8.27 \mathrm{E}-01$ & $4.10 \mathrm{E}+00$ & $480 \mathrm{k}$ & $6.04 \mathrm{E}-01$ & $6.30 \mathrm{E}+01$ \\
\hline $30 \mathrm{k}$ & $5.53 \mathrm{E}-01$ & $2.08 \mathrm{E}+00$ & $480 \mathrm{k}$ & 3.61E-01 & $2.33 \mathrm{E}+01$ \\
\hline $30 \mathrm{k}$ & 3.67E-01 & $1.18 \mathrm{E}+00$ & $480 \mathrm{k}$ & 2.39E-01 & $1.36 \mathrm{E}+01$ \\
\hline $66 \mathrm{k}$ & $1.23 \mathrm{E}+00$ & $2.33 \mathrm{E}+01$ & $480 \mathrm{k}$ & $1.59 \mathrm{E}-01$ & $8.78 \mathrm{E}+00$ \\
\hline $66 \mathrm{k}$ & $9.12 \mathrm{E}-01$ & $1.17 \mathrm{E}+01$ & $480 \mathrm{k}$ & $1.06 \mathrm{E}-01$ & $6.12 \mathrm{E}+00$ \\
\hline $66 \mathrm{k}$ & $6.96 \mathrm{E}-01$ & $7.02 \mathrm{E}+00$ & $480 \mathrm{k}$ & $6.65 \mathrm{E}-02$ & $4.28 \mathrm{E}+00$ \\
\hline $66 \mathrm{k}$ & $5.31 \mathrm{E}-01$ & $4.51 \mathrm{E}+00$ & $480 \mathrm{k}$ & $4.60 \mathrm{E}-02$ & $3.30 \mathrm{E}+00$ \\
\hline $66 \mathrm{k}$ & $4.08 \mathrm{E}-01$ & $3.16 \mathrm{E}+00$ & $480 \mathrm{k}$ & $3.06 \mathrm{E}-02$ & $2.43 \mathrm{E}+00$ \\
\hline $66 \mathrm{k}$ & $3.06 \mathrm{E}-01$ & $2.22 \mathrm{E}+00$ & $480 \mathrm{k}$ & $2.11 \mathrm{E}-02$ & $1.98 \mathrm{E}+00$ \\
\hline $66 \mathrm{k}$ & $2.33 \mathrm{E}-01$ & $1.69 \mathrm{E}+00$ & $480 \mathrm{k}$ & $1.40 \mathrm{E}-02$ & $1.53 \mathrm{E}+00$ \\
\hline $66 \mathrm{k}$ & $1.73 \mathrm{E}-01$ & $1.27 \mathrm{E}+00$ & $480 \mathrm{k}$ & $9.37 \mathrm{E}-03$ & $1.29 \mathrm{E}+00$ \\
\hline $66 \mathrm{k}$ & $1.31 \mathrm{E}-01$ & $9.84 \mathrm{E}-01$ & $480 \mathrm{k}$ & $6.25 \mathrm{E}-03$ & $1.00 \mathrm{E}+00$ \\
\hline $150 \mathrm{k}$ & $1.25 \mathrm{E}+00$ & $8.24 \mathrm{E}+01$ & $1 \mathrm{M}$ & $1.00 \mathrm{E}+00$ & $9.23 \mathrm{E}+02$ \\
\hline $150 \mathrm{k}$ & $1.02 \mathrm{E}+00$ & $4.62 \mathrm{E}+01$ & $1 \mathrm{M}$ & 7.19E-01 & $2.69 \mathrm{E}+02$ \\
\hline $150 \mathrm{k}$ & $7.87 \mathrm{E}-01$ & $2.52 \mathrm{E}+01$ & $1 \mathrm{M}$ & $5.53 \mathrm{E}-01$ & $1.97 \mathrm{E}+02$ \\
\hline $150 \mathrm{k}$ & $5.99 \mathrm{E}-01$ & $1.51 \mathrm{E}+01$ & $1 \mathrm{M}$ & $3.69 \mathrm{E}-01$ & $7.38 \mathrm{E}+01$ \\
\hline $150 \mathrm{k}$ & $4.54 \mathrm{E}-01$ & $9.64 \mathrm{E}+00$ & $1 \mathrm{M}$ & $2.75 \mathrm{E}-01$ & $4.32 \mathrm{E}+01$ \\
\hline $150 \mathrm{k}$ & $3.48 \mathrm{E}-01$ & $6.71 \mathrm{E}+00$ & $1 \mathrm{M}$ & $1.87 \mathrm{E}-01$ & $2.75 \mathrm{E}+01$ \\
\hline $150 \mathrm{k}$ & $2.74 \mathrm{E}-01$ & $5.00 \mathrm{E}+00$ & $1 \mathrm{M}$ & $8.84 \mathrm{E}-02$ & $1.07 \mathrm{E}+01$ \\
\hline $150 \mathrm{k}$ & $1.84 \mathrm{E}-01$ & $2.91 \mathrm{E}+00$ & $1 \mathrm{M}$ & $5.89 \mathrm{E}-02$ & $7.83 \mathrm{E}+00$ \\
\hline $150 \mathrm{k}$ & $1.38 \mathrm{E}-01$ & $2.21 \mathrm{E}+00$ & $1 \mathrm{M}$ & $3.93 \mathrm{E}-02$ & $5.97 \mathrm{E}+00$ \\
\hline $150 \mathrm{k}$ & $1.06 \mathrm{E}-01$ & $1.85 \mathrm{E}+00$ & $1 \mathrm{M}$ & $2.68 \mathrm{E}-02$ & $4.66 \mathrm{E}+00$ \\
\hline $150 \mathrm{k}$ & $7.98 \mathrm{E}-02$ & $1.44 \mathrm{E}+00$ & $1 \mathrm{M}$ & $1.85 \mathrm{E}-02$ & $3.90 \mathrm{E}+00$ \\
\hline $150 \mathrm{k}$ & $6.42 \mathrm{E}-02$ & $1.27 \mathrm{E}+00$ & $1 \mathrm{M}$ & $1.12 \mathrm{E}-02$ & $2.88 \mathrm{E}+00$ \\
\hline $150 \mathrm{k}$ & 4.89E-02 & $1.07 \mathrm{E}+00$ & $1 \mathrm{M}$ & 7.73E-03 & $2.39 \mathrm{E}+00$ \\
\hline $150 \mathrm{k}$ & $3.81 \mathrm{E}-02$ & $9.10 \mathrm{E}-01$ & $1 \mathrm{M}$ & 4.87E-03 & $1.80 \mathrm{E}+00$ \\
\hline $271 \mathrm{k}$ & $1.24 \mathrm{E}+00$ & $1.73 \mathrm{E}+02$ & $1 \mathrm{M}$ & $3.04 \mathrm{E}-03$ & $1.43 \mathrm{E}+00$ \\
\hline $271 \mathrm{k}$ & $8.16 \mathrm{E}-01$ & $5.21 \mathrm{E}+01$ & $1 \mathrm{M}$ & $1.98 \mathrm{E}-03$ & $1.08 \mathrm{E}+00$ \\
\hline
\end{tabular}

